\title{
Optimal Control Strategy for Hepatitis B Virus Epidemic in Areas of High Endemicity
}

\author{
IJALANA, Clara O. ${ }^{1}$; YUSUF, Tajudeen T. ${ }^{2}$ \\ ${ }^{1}$ Department of Mathematical Sciences, Federal University of Technology, Akure, Nigeria \\ ${ }^{2}$ Department of Mathematical Sciences, Federal University of Technology, Akure, Nigeria
}

*Corresponding Author: YUSUF, Tajudeen T., Department of Mathematical Sciences, Federal University of Technology, Akure, Nigeria

\begin{abstract}
Hepatitis B virus infection remains endemic in some parts of northern Nigeria despite the availability of a potent vaccine against the infection. There is therefore the need for continuous efforts to eradicate the disease in order to forestall its spread to other parts of the country, more so that the disease highly infectious. We present an improved deterministic model to describe the spread of Hepatitis $B$ virus infection in areas of high endemicity using vaccination of the susceptibles and early detection cum effective treatment of acute Hepatitis B virus infected individuals as control measures. We formulate an optimal control problem subject to the model dynamics incorporating the two control measures with the goal of finding the optimal combination of the two control measures that will minimize the cost of implementing the control measures as well as reduce the incidences and prevalence of the disease. Using Pontryagin's maximum principle, we obtain the optimality system and solve the system numerically. Our simulations shows that starting treatment for acutely infected individuals early enough and improving the potency of the vaccines reduces the incidence and prevalence of Hepatitis B virus infection drastically within ten years.
\end{abstract}

Keywords: Hepatitis B Virus, Vaccination, Early treatment, Pontryagin's maximum principle, Optimality system, Disease prevalence, Disease incidences.

\section{INTRODUCTION}

Hepatitis B virus infection is one of the major health problems in the world today with over one-third of the world's population (over two billion people) acutely infected with Hepatitis-B Virus; more than 240 million people are chronically infected by the virus. About 780,000 people die yearly as a result of the infection. Worse still, about 24-40 percent of the chronically infected carriers die from liver cirrhosis or Hepatocellular Carcinoma (HCC) a leading cause of cancer-related death worldwide and the burden of this devastating cancer is expected to increase further in coming years $[13,15]$. Infact, hepatocellular cancer (HCC) alone accounted for over 500,000 deaths per year, making it the third leading cause of cancer mortality worldwide $[1,10,11]$.

Usually, people living with Hepatitis B virus infection can fight off the infection within few months and develop a life-long immunity (this evidence has been shown through blood tests with an evidence of immunity and no signs of an active infection) [14].

Most people got infected early in life through perinatal transmission or during childhood and many of them are unaware they had been infected since they show no clinical symptoms and do not feel sick, so they can successfully transmit the virus to susceptible individual unconsciously. Carriers of this viral infection may not show any symptom of the disease even though they had been infected by the virus unknowingly and this made it more dangerous. Moreso, HBV is 50 times more contagious than

HIV [5]. Hence, it is imperative for individuals to protect themselves.

The outcome of HBV infection varies greatly from person to person. In most of the cases, the infection is cleared spontaneously. However, 5\% - 10\% of infected adults develop chronic infection. By contrast, $40 \%$ - 90\% of children who are born to HBV-infected mothers will progress to develop a persistent liver disease. Children and adolescents are in greater risks than adults because adults upon exposure may show some clinical symptom and have about $90 \%$ chance of been acutely infected with 
clinical symptoms immediately after infection but do not completely clear the virus and some of them become chronically infected. About $25 \%$ of chronic carriers die of liver cancer. Thus, Hepatitis B is one of the most common viral sources of cancer in the world today and also the most important carcinogen with Tobacco as the second [5].

Timely interventions also play a vital role in managing the progression of the infection in a patient and in controlling its spread in the society. For example, the incidence of Ebola Virus in Nigeria in 2014 was eradicated due to timely intervention such as quarantine and screening of individuals that may have been exposed to the virus. Most diseases will remain endemic due to lack of adequate policies and timely interventions. In the past, some diseases that were previously endemic in some places have been eradicated in many areas of the world. Examples are Malaria, Small pox, Rubella, etc $[2,19]$.

Mathematical models are vital tools in understanding the dynamics of any epidemic and results obtained from such models further assist health policy makers to develop various control measures and preventive policies to avert incidences of infectious diseases. In addition, optimal control theory is another powerful mathematical tool used in decision making for situations involving complex health or biological situations, Lenhart and Workman [9]. For example, Seth and Staats [12] formulated control problem subject to a simple deterministic epidemic models using levels of vaccination programmes as control measures.

Bakare et al. [3] developed an SIR epidemic model with constant recruitment using educational campaign and treatment strategies as controls to minimize the total number of infective individuals and the cost associated with the use of the educational campaign and treatment. Their model due to its dynamical behavior is suitable for diseases like H1N1 (influenza), measles, chicken pox, mumps, etc.

In a related study, Yusuf and Benyah [17] considered an SIR model with variable population size using vaccination and treatment as control strategies. They applied optimal control theory to optimally combine the two control measures. They concluded that the optimal way to control or drive an epidemic to extinction within a specific period of time is to use more of vaccination strategy (whenever it is available) and less of treatment as it is more cheaper and less risky to prevent the occurrence of an infection than to cure it.

Behncke [4] studied the application of optimal control theory on a simple SIR epidemiological model which incorporates vaccination, quarantine and health promotion campaign as controls. Greenhalgh [8] considered the control of the spread of an epidemic in a homogeneously mixed population controlled by vaccination of susceptibles and isolation of infected. However, for case of controlling the spread of infections in a heterogeneously mixed population, he found that the optimal control strategy (vaccination policy) is linked to the changing growth rate.

Yusuf and Benyah [19] applied optimal control strategy for controlling the spread of HIV/AIDS in South Africa. They considered a deterministic model for the spread of HIV with a removed class made up of individuals who have sufficiently modified their sexual habits and they are therefore considered to be immune to the virus infection through sexual contacts. Their research work generally centered on finding an optimal strategy to minimize the cost of implementing combined control programme as well as the incidences of the disease. Their findings shows that the optimal way to mitigate the spread of the disease is for susceptible individuals to consistently practice a safe sex while ARV treatment should be initiated for infected individuals as soon as they progress to pre-AIDS stage. Though, this strategy may appear expensive; its effect will positively benefit the society and the HIV/AIDS individuals in the long run.

In 2014, Yusuf et al. [18] considered a model for the spread of HBV and used the model to evaluate the impact of strategies that includes changes in sexual habits and vaccination on the prevalence and incidence of HBV. Also, they evaluated investment (financial budget) in controlling the prevalence of the disease and they found that in places of high endemicity, the impact of investing in creating awareness and introduction of vaccine will effectively reduce the level of prevalence of the infection.

Wiah et al. [16] used a non-linear extended deterministic model for accessing the impact of immigration on the spread of HBV infection in a population with acute and chronic HBV infected individuals. The impact of optimal control of the treatment and vaccination strategy on the transmission dynamics of the disease in a population with constant immigration and homogeneous 
mixing is undeniably of great importance. Vaccination would not be cost effective in the population if the cost associated with preventing a small group of new infections in an adult community outweighs the cost of immunizing a large population and the cost of existing untreated chronic HBV infections. Thus, the treatment strategy was always more cost effective to implement than vaccination.

However, it is important to state here that, the conclusion from this work cannot be generalized for all cases of controlling the spread of infectious disease, even HBV. Thus, each infectious disease and the prevailing epidemic situation in the location of interest may determine which combination of control measures would be optimally effective in curtailing the spread and containing an infectious disease.

This paper is structured as follows: In section 2, we formulate the model for HBV transmission dynamics. In section 3, we reformulated the modeled situation as an optimal control problem and obtained the needed optimality system using Pontryagin's maximum principle. In section 4, we numerically solve the resulting optimality system and simulate our results for three scenarios of interest. We also discuss the results from the simulations.

\section{MODEL FORMULATION}

The model sub-divides the total population at time $t$ denoted as $\mathrm{N}(\mathrm{t})$ into five epidemiological classes representing the susceptible $S(t)$, the exposed $E(t)$, the acute infection $A(t)$, the chronic $C(t)$ and the recovered $\mathrm{R}(\mathrm{t})$. Thus,

$$
N(t)=S(t)+E(t)+A(t)+C(t)+R(t)
$$

Individuals enters the susceptible population either birth or immigration at the constant rate $\Lambda$. The susceptible population decreases due to infection of the susceptibles by the infected individuals at the rate $\beta$. The susceptible class is further reduced due to natural death at the rate $\mu$ and vaccination of susceptible individuals leaving the group at the rate $u_{1}$. Thus,

$$
\frac{d S}{d t}=\Lambda-\frac{\beta S}{N}(A+\alpha C)-u_{1} S-\mu S
$$

Based on the physiology of Hepatitis B virus, there are three stages of HBV infection represented by the epidemiological classes $E, A$, and $C$. So, as soon as a susceptible individual is infected with HBV, he/she becomes a member of the exposed class $E$ at the rate $\beta$. Individuals in the exposed class could progress from the latent HBV stage to the acute Hepatitis B virus infection stage at the rate $\rho_{1}$ or naturally recover from the infection at rate $\delta$ or die due to natural death at rate $\mu$. Hence,

$$
\frac{d E}{d t}=\frac{\beta S}{N}(A+\alpha C)-\gamma E-\rho_{1} E-\mu E
$$

The population of the acute class is increased by individuals whose infection did not clear off after a short infectious period at the rate $\rho_{1}$. However, acute-HBV infected individuals undergoing treatment do not recover completely from the infection, thus they could eventually progresses to the chronic stage of the infection (since there is no permanent cure for the disease). Therefore, the Acute-HBV infected population decreases due to progression from the acute-infected class and due to natural death at rate $\mu$. So,

$$
\frac{d A}{d t}=\rho_{1} E-\left(1-u_{2}\right) \rho_{2} A-\mu A
$$

The population of the chronic class with clinical symptoms increases at the rate $\rho_{2}$ due to the disease progression from the acute stage while it decreases as a result of deaths which could disease induced or natural at the rates $\delta$ and $\mu$ respectively. Thus,

$$
\frac{d C}{d t}=\left(1-u_{2}\right) \rho_{2} A-\mu C-\delta C
$$

The population of the recovered class increases following vaccination of individuals in the susceptible class at rate $u_{1}$ and natural recovery of exposed llatent class individuals the rate $\gamma$ while individuals in this class die at rate $\mu$. Thus, 


$$
\frac{d R}{d t}=u_{1} S+\gamma E-\mu R
$$

Summarily, the model for the transmission dynamics of Hepatitis B virus is given by the following system of deterministic coupled differential equations:

$$
\begin{aligned}
& \frac{d S}{d t}=\Lambda-\frac{\beta S}{N}(A+\alpha C)-u_{1} S-\mu S \\
& \frac{d E}{d t}=\frac{\beta S}{N}(A+\alpha C)-\gamma E-\rho_{1} E-\mu E \\
& \frac{d A}{d t}=\rho_{1} E-\left(1-u_{2}\right) \rho_{2} A-\mu A \\
& \frac{d C}{d t}=\left(1-u_{2}\right) \rho_{2} A-\mu C-\delta C \\
& \frac{d R}{d t}=u_{1} S+\gamma E-\mu R
\end{aligned}
$$

However, we derived the disease basic reproduction number $\mathrm{R}_{0}$ using the next generation matrix approach as:

$$
\mathrm{R}_{0}=\frac{\beta \mu \rho_{1}}{\left(\rho_{1}+\mu+\gamma\right)\left(\left(1-\mathrm{u}_{2}\right) \rho_{2}+\mu\right)\left(\mu+\mathrm{u}_{1}\right)}+\frac{\beta \mu \rho_{1} \rho_{2} \alpha\left(1-\mathrm{u}_{2}\right)}{\left(\rho_{1}+\mu+\gamma\right)\left(\left(1-\mathrm{u}_{2}\right) \rho_{2}+\mu\right)\left(\mu+\mathrm{u}_{1}\right)(\mu+\delta)}
$$

This is an important threshold quantity to demonstrate that the situation under consideration is an endemic one (i.e. a case where $\mathrm{R}_{0}>1$ ).

\section{Optimal Control Problem Formulation}

We define our objective functional as

$$
\mathbf{J}=\min _{\left(u_{1}, u_{2}\right)} \int_{t_{0}}^{t_{1}}\left(\frac{1}{2} w_{1} u_{1}{ }^{2}+\frac{1}{2} w_{2} u_{2}{ }^{2}+w_{3} S+w_{4} A+w_{5} C\right) d t
$$

subject to the state equations (2.6) with appropriate state initial conditions, $t_{0}$ is the initial time and $t_{1}$ is the terminal time, while the control set $\boldsymbol{U}$ whose elements are Lebesque measurable is defined as

$$
\boldsymbol{U}=\left\{\left(\mathrm{u}_{1}, \mathrm{u}_{2}\right) \mid 0 \leq \mathrm{u}_{1} \leq \mathrm{u}_{1_{\max }}, 0 \leq \mathrm{u}_{2} \leq \mathrm{u}_{2_{\max }}\right\} .
$$

and the $w_{1}, w_{2}, w_{3}, w_{4}, w_{5}$ are the relative weight constants which balances each term in the integrand to reduce the dominance of any of the term in the integral. The weight constants $w_{1}$ and $w_{2}$ measures the cost or effort required for the implementation of each of the two control measures adopted while $w_{3}, w_{4}$ and $w_{5}$ measures the relative importance of reducing the associated classes on the spread of the disease. The control $\mathrm{u}_{1}$ represents the proportion of susceptible individuals that is effectively vaccinated and hence have full immunity per unit time, while $u_{2}$ is the proportion of individuals in the acute class who are currently being effectively managed so that their case do not deteriorate into the chronic stage. Hypothetically, we set $\mathrm{u}_{1_{\max }}=0.9$ andu $_{2_{\max }}=0.5$.

\subsubsection{Existence of an Optimal Control Pair}

Here, we examine the sufficient conditions for the existence of a solution to the optimal control problem.

\section{Theorem 3.0.1}

There exists an optimal control set $\mathrm{u}_{1}{ }^{*}, \mathrm{u}_{2}{ }^{*}$ with a corresponding solution $\left(\mathrm{S}^{*}, \mathrm{E}^{*}, \mathrm{~A}^{*}, \mathrm{C}^{*} \mathrm{R}^{*}\right)$ to the model system that minimizes $J\left(u_{1}, u_{2}\right)$ over $\mathbf{U}$.

Proof

Based on the Flemming and Rishel theorem [6], we establish the existence of the optimal control by showing the compactness of the control and the state space and that the stated problem is convex and bounded. The Flemming and Rishel theorem are as highlighted below: 
$i$. The sets of all solutions of the state equations and its associated initial controls with the corresponding control function in $\boldsymbol{U}$ is nonempty.

ii. $\quad$ The state system can be written as a linear function of the control variables with coefficients dependent on time and the state variables.

iii. $\quad$ The integrand of the objective functional (3.1) is convex on $\boldsymbol{U}$ and it satisfies

$$
\boldsymbol{K} \geq w\left\|u_{1}, u_{2}\right\|^{2}-w_{1} u_{1}^{2}+w_{2} u_{2}^{2}+w_{3} S+w_{4} E+w_{5} A
$$

where

$$
w>0, w_{1}>0 \text { and } \boldsymbol{K}=\frac{1}{2}\left(w_{1} u_{1}^{2}+w_{2} u_{2}^{2}\right)+w_{3} S+w_{4} E+w_{5} A
$$

Let the integrand beK. In order to prove the above stated theorem, it suffices to show that $\boldsymbol{K}$ is bounded and convex. Using the approach of Yusuf and Benyah [19], if the solutions to the states equations in (2.6) are a priori bounded and if the state equations are continuous and Lipschitz in the state variables, then $\exists$ a unique solution corresponding to the controlsu $u_{1}, u_{2} \in \boldsymbol{U}$. Since $(S, E, A, C, R) \in \Omega$, then all the state variables of the model (2.6) are bounded (i.e bounded below and above). Thus, the system is Lipschitz with respect to the state equations hence, the condition (1) holds. In addition, the state equations are linearly dependent on the controls $u_{1}, u_{2}$ therefore the condition (2) also holds.

In order to establish condition (3), we observe that the integrand $\boldsymbol{K}$ is convex because of the quadratic nature of the control term. In addition,

$$
\begin{aligned}
& \boldsymbol{K}=\frac{1}{2}\left(w_{1} u_{1}{ }^{2}+w_{2} u_{2}{ }^{2}\right)+w_{3} S+w_{4} E+w_{5} A \\
& \geq \frac{1}{2}\left(w_{1} u_{1}^{2}+w_{2} u_{2}^{2}\right) \\
& \text { since } w_{i}>0, \quad i=1,2, \ldots, 5 \\
& \geq \frac{1}{2}\left(w_{1} u_{1}^{2}+w_{2} u_{2}^{2}\right)-w_{1} \\
& \text { since } w_{1} u_{1}^{2}-w_{1}<0 \text {, } \\
& \geq \min \left(\frac{1}{2} w_{1}, \frac{1}{2} w_{2}\right)\left(u_{1}^{2}+u_{2}^{2}\right)-w_{1} \text {, } \\
& \geq w\left\|u_{1}, u_{2}\right\|^{2}-w_{1}, \\
& \text { where } w=\min \left(\frac{1}{2} w_{1}, \frac{1}{2} w_{2}\right) \text {. }
\end{aligned}
$$

This establishes the fact that $\boldsymbol{K}$ is bounded. Hence, we have a unique solution of the optimality system for any small time interval. Moreover, the uniqueness of the solution of the optimality system guarantees the uniqueness of the optimal control if it exists.

\subsection{Characterization of the Optimal Control pair $\left(u_{1}{ }^{*}, u_{2}{ }^{*}\right)$}

We characterize the optimal control pair $\left(u_{1}{ }^{*}, u_{2}{ }^{*}\right)$ for the two control measures adopted and the corresponding states $\left(S^{*}, E^{*}, A^{*}, C^{*}, R^{*}\right)$. The necessary conditions for an optimal control pair are obtained using Pontryagin's maximum principle (PMP) [9].

Theorem 3.1 Necessary Conditions for the existence of an Optimal control pair

Let $u_{1}{ }^{*}, u_{2}{ }^{*} \in \boldsymbol{U}$ be an optimal control pair with the corresponding states $\left(S^{*}, E^{*}, A^{*}, C^{*}, R^{*}\right)$, then there exists the adjoint variables $\lambda_{i}$ for $i=1,2, \cdots, 5$ satisfying

$$
\begin{aligned}
& \lambda_{1}{ }^{\prime}=-w_{5}+\lambda_{1}\left[\frac{\beta N(A+\alpha C)+\beta S(A+\alpha C)}{N^{2}}\right]+\left(u_{1}{ }^{*}+\mu\right) \\
& \quad-\lambda_{2}\left[\frac{\beta N(A+\alpha C)+\beta S(A+\alpha C)}{N^{2}}\right]-\lambda_{5} u_{1}{ }^{*},
\end{aligned}
$$




$$
\begin{aligned}
& \lambda_{2}{ }^{\prime}=-\lambda_{1}\left[\frac{\beta S(A+\alpha C)}{N^{2}}\right]-\lambda_{2}\left[\frac{\beta S(A+\alpha C)}{N^{2}}-\left(\rho_{1}+\mu+\gamma\right)\right]-\lambda_{3} \rho_{1}+\lambda_{5} \gamma \\
& \lambda_{3}{ }^{\prime}=-w_{3}+\lambda_{1}\left[\frac{\beta S N+\beta S(A+\alpha C)}{N^{2}}\right]-\lambda_{2}\left[\frac{\beta N S+\beta S(A+\alpha C)}{N^{2}}\right] \\
& +\lambda_{3}\left[\left(1-u_{2}{ }^{*}\right) \rho_{2}+\mu\right]-\lambda_{4}\left[\left(1-u_{2}{ }^{*}\right) \rho_{2}\right] \\
& \lambda_{4}{ }^{\prime}=-w_{4}+\lambda_{1}\left[\frac{\beta S N \alpha+\beta S(A+\alpha C)}{N^{2}}\right]-\lambda_{2}\left[\frac{\beta N \alpha S+\beta S(A+\alpha C)}{N^{2}}\right]+\lambda_{4}[\mu+\delta] \\
& \lambda_{5}{ }^{\prime}=-\lambda_{1}\left[\frac{\beta S(A+\alpha C)}{N^{2}}\right]+\lambda_{2}\left[\frac{\beta S(A+\alpha C)}{N^{2}}\right]+\lambda_{5} \mu
\end{aligned}
$$

and the transversality conditions

$$
\lambda_{1}\left(t_{f}\right)=\lambda_{2}\left(t_{f}\right)=\cdots, \lambda_{5}\left(t_{f}\right)=0
$$

as the terminal conditions at the final time $t_{f}$ while the optimal control variables are defined as:

$$
\begin{aligned}
& u_{1}^{*}=\min \left\{\max \left(0, \frac{S\left(\lambda_{1}-\lambda_{5}\right)}{w_{1}}, u_{1_{\max }}\right)\right\} \\
& u_{2}{ }^{*}=\min \left\{\max \left(0, \frac{A\left(\lambda_{4} \rho_{2}-\lambda_{3} \rho_{2}\right)}{w_{2}}, u_{2_{\max }}\right)\right\} .
\end{aligned}
$$

Proof

Using the Pontryagin's maximum principle (PMP), we obtain Equation (3.1.1) from

$$
\lambda_{1}{ }^{\prime}=-\frac{\partial \mathbf{H}}{\partial S}, \lambda_{1}{ }^{\prime}=-\frac{\partial \mathbf{H}}{\partial E}, \lambda_{1}{ }^{\prime}=-\frac{\partial \mathbf{H}}{\partial A}, \lambda_{1}{ }^{\prime}=-\frac{\partial \mathbf{H}}{\partial C}, \lambda_{1}{ }^{\prime}=-\frac{\partial \mathbf{H}}{\partial R}
$$

where the Hamiltonian $\mathbf{H}$ is given by

$$
\begin{aligned}
\mathcal{H} & =\frac{1}{2} w_{1} u_{1}{ }^{2}+\frac{1}{2} w_{2} u_{2}{ }^{2}+w_{3} A 1 w_{4} C+w_{5} S+\lambda_{1}\left[\Lambda-\frac{\beta S}{N}(A+\alpha C)-u_{1} S-\mu S\right] \\
& +\lambda_{2}\left[\frac{\beta S}{N}(A+\alpha C)-\gamma E-\rho_{1} E-\mu E\right]+\lambda_{3}\left[\rho_{1} E-\left(1-u_{2}\right) \rho_{2} A-\mu A\right] \quad m \\
& +\lambda_{4}\left[\left(1-u_{2}\right) \rho_{2} A-\mu C-\delta C\right]+\lambda_{5}\left[u_{1} S+\gamma E-\mu R\right]
\end{aligned}
$$

Using the transversality condition of the PMP yields

$$
\lambda_{1}\left(t_{f}\right)=\lambda_{2}\left(t_{f}\right)=\cdots \lambda_{5}\left(t_{f}\right)=0
$$

Also, solving for $u_{1}{ }^{*}, u_{2}{ }^{*}$ based on the optimality condition of the PMP gives:

$$
\begin{aligned}
& u_{1}^{*}=\frac{S\left(\lambda_{1}-\lambda_{5}\right)}{w_{1}}, \\
& u_{2}{ }^{*}=\frac{A\left(\lambda_{3} \rho_{2}-\lambda_{5} \rho_{2}\right)}{w_{2}}
\end{aligned}
$$

respectively. By imposing the bounds $0 \leq u_{1} \leq u_{1_{\max }}$ and $0 \leq u_{2} \leq u_{2_{\max }}$ on the control variables, we have 


$$
\begin{gathered}
u_{1}^{*}=\min \left\{\max \left(0, \frac{S\left(\lambda_{1}-\lambda_{5}\right)}{w_{1}}\right), u_{1 \max }\right\} \\
u_{2}{ }^{*}=\min \left\{\max \left(0, \frac{A\left(\lambda_{3} \rho_{2}-\lambda_{5} \rho_{2}\right)}{w_{2}}\right), u_{2 \max }\right\} .
\end{gathered}
$$

Thus, the required optimality system consists of the state variables system of equations (2.6) with its initial conditions and the adjoint variables system of equations (3.1.1) with its terminal conditions which incorporates the optimal control variables characterization in its dynamics.

\section{Simulation Results AND Discussion}

We numerically solve the resulting optimality system using Runge Kutta fourth order scheme by adopting Forward--Backward Sweep approach. This approach solves the state equations forward in time with an initial guess for $\mathrm{u}_{1}$ and $\mathrm{u}_{2}$ while the adjoint equations are solved backward in time. Thereafter, the control variables are updated based on equations (3.1.10). This procedure is done iteratively until convergence is achieved.

\subsection{Data}

The table (4.1) specifies the parameter values and table (4.2) indicates the initial conditions for each of the state variables used in carrying out our simulation.

Table 4.1. The model parameter description and their corresponding values

\begin{tabular}{|c|l|l|c|}
\hline PARAMETER & DEFINITION & VALUE & REFERENCE \\
\hline$\Lambda$ & Recruitment rate & 2.0 & Estimate \\
\hline$\mu$ & Natural death rate & 0.002 & Estimate \\
\hline$\delta$ & HBV induced death rate & 2.5 & Estimate \\
\hline$\rho_{1}$ & Movement rate from Exposed to Acute & 6 per year & {$[5]$} \\
\hline$\rho_{2}$ & Movement rate from Acute to Chronic & 4 per year & {$[5]$} \\
\hline$\alpha$ & Transmission multiplier & 3.0 & {$[5]$} \\
\hline $\mathrm{u}_{1}$ & Control attached to vaccination & $0-1$ & {$[5]$} \\
\hline $\mathrm{u}_{2}$ & Control based on treatment & $0-1$ & {$[5]$} \\
\hline$\gamma$ & Recovery rate due to immunity & 0.025 per year & {$[5]$} \\
\hline$\beta$ & Disease transmission rate & 0.95 & Estimate \\
\hline
\end{tabular}

Table 4.2. Initial Conditions for the model's state variables

\begin{tabular}{|l|c|c|c|c|c|}
\hline VARIABLES & Susceptibles S(0) & $\begin{array}{c}\text { Exposed } \\
\mathrm{E}(0)\end{array}$ & $\begin{array}{c}\text { Acute } \\
\mathrm{A}(0)\end{array}$ & $\begin{array}{c}\text { Chronic } \\
\mathrm{C}(0)\end{array}$ & $\begin{array}{c}\text { Recovered } \\
\mathrm{R}(0)\end{array}$ \\
\hline VALUES & 92.5 & 2.0 & 3.0 & 1.5 & 1.0 \\
\hline
\end{tabular}

It is important to emphasize here that the set of parameter values above yields $R_{0}=1.45>1$ which implies that $\mathrm{HBV}$ is endemic in the scenario under consideration. Also, the initial condition for the state variables is a realistic hypothetic one. We simulate the optimality system with parameter values from Table 3.1 using the weight constants $w_{1}=1.0, w_{1}=10.0$ and we vary the weights for $w_{3}, w_{4}$ and $w_{5}$ depending on the scenario being considered.

Assuming that there are adequate resources to implement the following maximum effectiveness levels of the control $u_{1}=0.9, u_{2}=0.5$, we considered the following three scenarios:

(i) The scenario with Vaccination ONLY $\left(w_{3}=1.0, w_{4}=100.0, w_{5}=1.0\right)$

(ii) The scenario with Treatment of the Acute HBV infected ONLY $\left(w_{3}=1000.0, w_{4}=\right.$ 1.0, $w 5=1.0$

(iii) The scenario of combining BOTH Vaccination and Treatment $\left(w_{3}=1.0, w_{4}=\right.$ 1.0, $w 5=1.0$ 


\subsection{Numerical results}

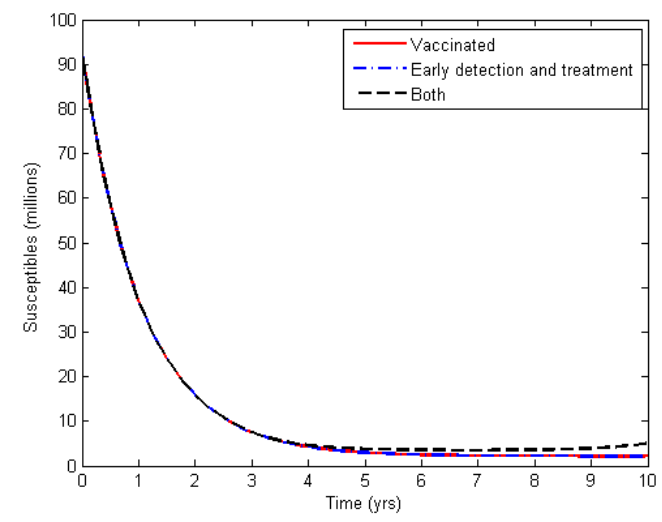

Fig4.1Population Profile for the S-class

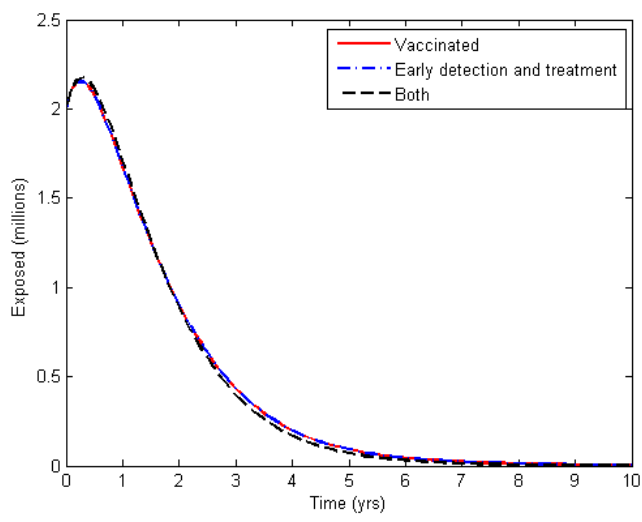

Fig 4.2 Population Profile for the E-class

Fig 4.1 shows the population profile of the susceptible class which is the largest of the five subpopulations. It is assumed that not all individuals were vaccinated at birth, thus we make 92.50 million of the entire 100million populations to be susceptible to the disease. From the fig 4.1, we observed that the impact of the two control measures and their combination plays a significant role in lowering the level of the population susceptibility significantly as time progresses. In less than three years, the susceptible population is reduced to about a ninth of its initial population as a result of the implementation of the control measures.

By the fifth year, if the implementation of these control measures is adequately carried out, then the entire population will eventually come to a point where the population of the susceptible individuals in the population is minimal. This will reduce new cases of the disease drastically because there will be fewer people that can be infected. Thus, this could lead to eventual eradication of the disease in the long run.

Fig. 4.2 shows the population profile of the Exposed individuals in the entire population. At the initial stage, the population rises from 2.0 million to about 2.2 million but as the time progresses, the population of the exposed for the three scenarios reduces drastically till it varnishes. Though, application of both controls concurrently reduces the population of the Exposed HBV class most.

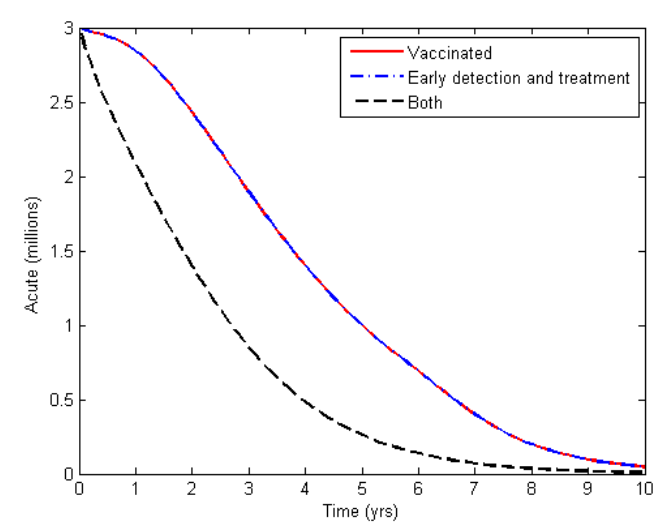

Fig 3.3 Population Profile for the A-class

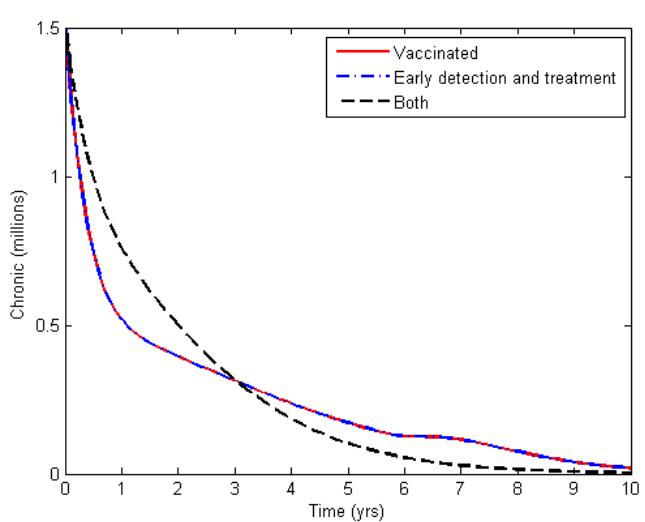

Fig 3.4 Population Profile for the C-class

Fig. 4.3 shows the population profile of the acute HBV-infected class with respect to the three scenarios considered. In the first year, deploying early detection cum treatment only reduces the population to about 2.85 million while both vaccination and treatment reduces to about 2.1 million.

In the second year, vaccination only and treatment only reduces the acute infected HBV class population to 2.4 million while the combination of both in the second year reduces the class population to 1.4 million. Thus, using the two control measures concurrently is more effective in controlling the Acute HBV-infected population. As time progresses, the population eventually reduces to less than 0.1 million as a result of combining both controls. 
Fig. 4.4 shows the population profile of chronic HBV-infected class with respect to the three scenarios considered. The graph shows that the strategy of treatment only or vaccination only performs better in the first three years of implementation while the control strategy which combines both controls does better in the latter years. This implies that combining both controls concurrently will be more effective in the long-run.

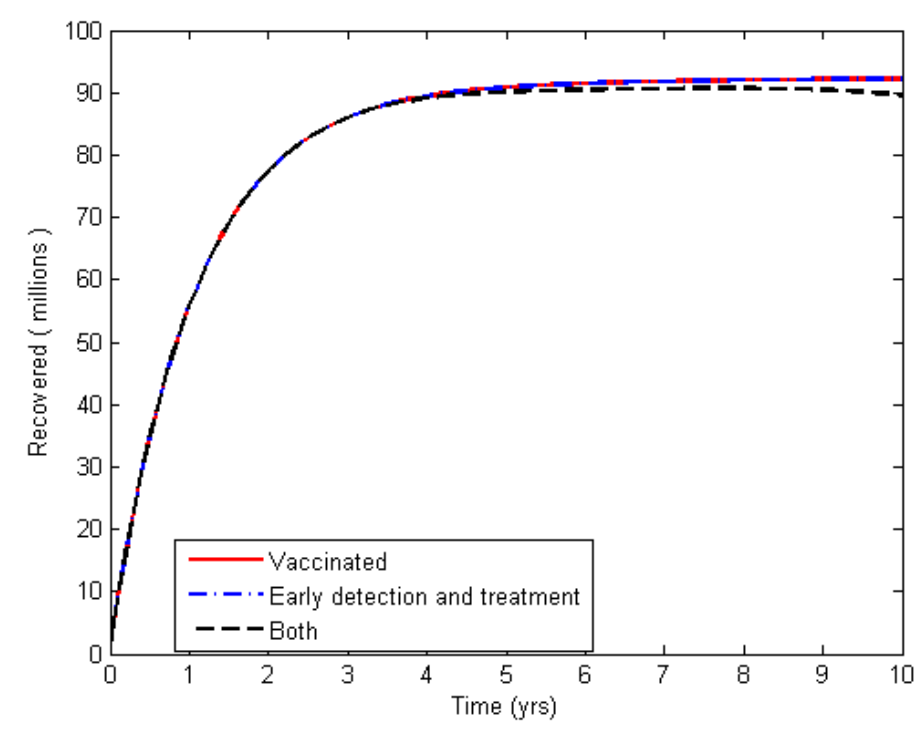

Fig 4.5 Population Profile for the R-class

Fig. 4.5 shows the population profile of the recovered class with respect to the three scenarios considered. Here, the results show that the population in the recovered class increases over time for all the scenarios though population for the scenario of vaccination only and that treatment only were higher after the fifth year. However, the difference in the population profile of the first two scenarios as compared to the third scenario is not too substantial. In general, these graphs show that the population in the recovered class will continue to increase over time until it reaches equilibrium where it stabilizes. At this point, the spread of HBV in the community will be minimal.

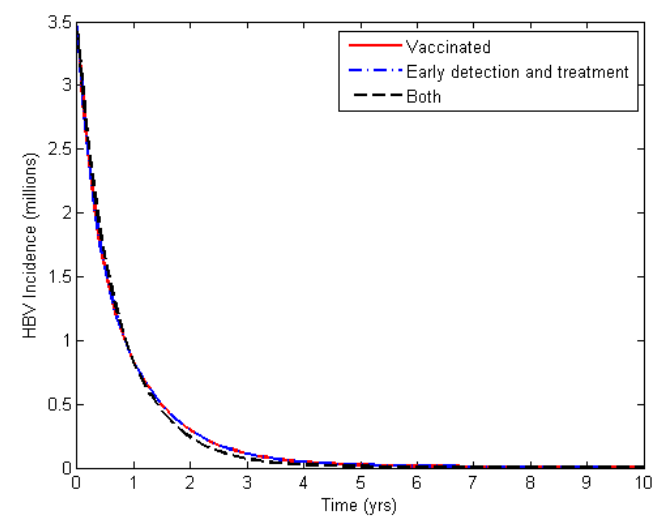

Fig. 4.6. Incidence of $H B V$

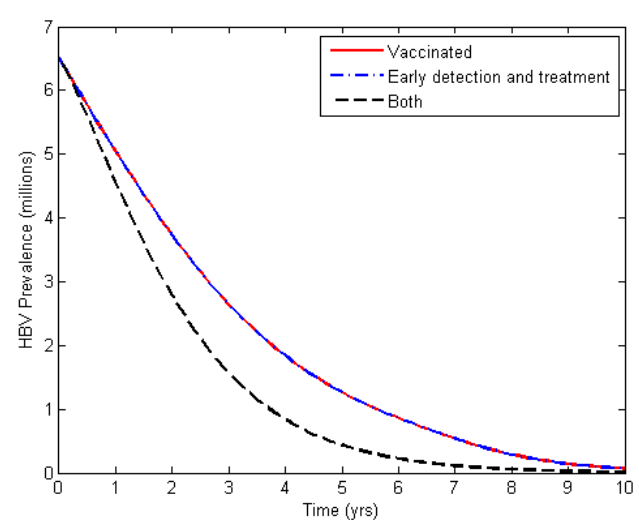

Fig. 4.7. Prevalence of $H B V$

Fig. 4.6 shows new cases of the HBV infection. We observed that either of the controls deployed will work effectively in reducing the occurrences of new cases of HBV infection but employing both controls concurrently will be more effective in reducing the incidence of HBV infection. Figure 4.7 shows the level of prevalence of HBV infection in the community for the three scenarios considered. We observed that combination of both Vaccination and early detection cum treatment contributes immensely in the reduction of HBV spread within the community. This suggests that the application of vaccination and treatment should be employed together in other to forestall the prevalence of the disease. 


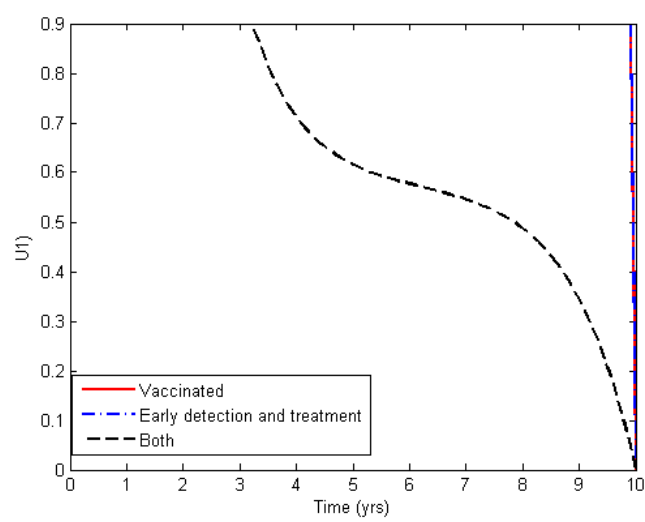

Fig. 4.8 Profile for $u_{1}$ (Vaccination)

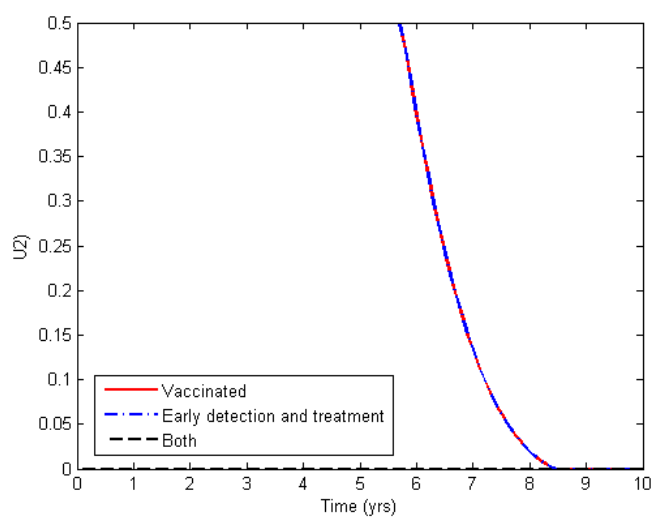

Fig. 4.9 Profile for $u_{2}$ (Treatment)

Fig. 4.8 shows the minimum success rate of the vaccination programme per unit time that is required to achieve earlier presented level of incidence and prevalence of the disease. The graph shows that for the case where both measures were considered, the success rate required was significantly lower, compared to the case when each measure was implemented alone. Fig. 4.9 shows the minimum effective treatment rate per unit time that must be achieved in order to record the level of incidence and prevalence of the disease presented in figs. 4.6 and 4.7 respectively. We observe that the effective treatment success rate remains low for the case of treatment only and both while it was high for the scenario with vaccination.

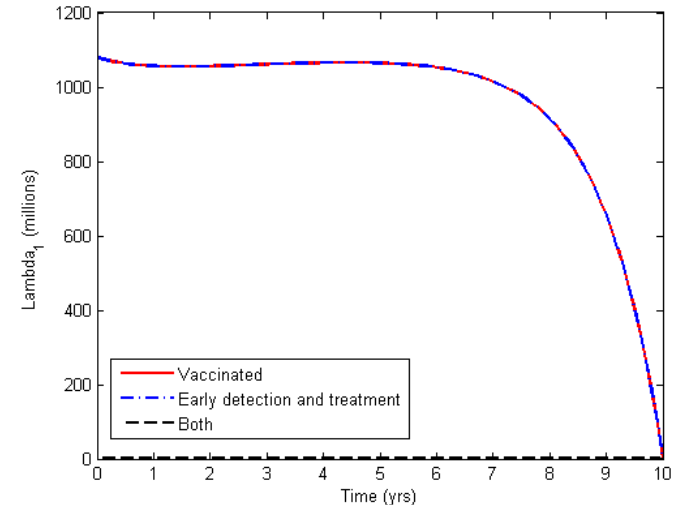

Fig. 4.10 Adjoint variable $\lambda_{1}$

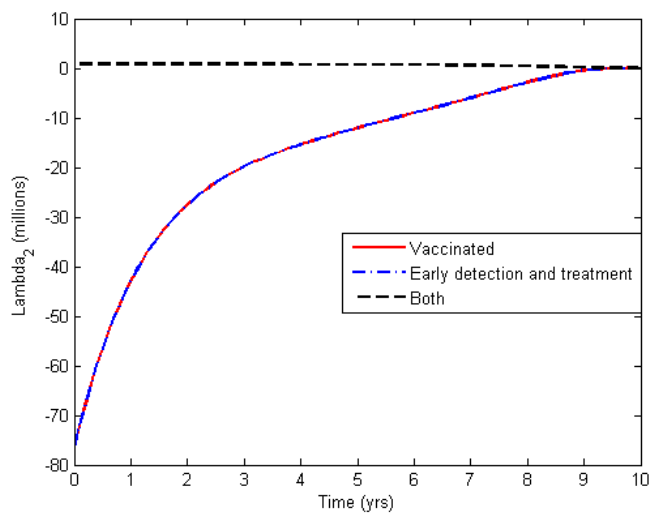

Fig. 4.11 Adjoint variable $\lambda_{2}$

Figs. 4.11, 4.12 and 4.13 shows that there is a zero, or negative marginal cost associated with a unit change in the exposed class population, Acute HBV-infected population, and chronic HBV-infected population respectively. The negative cost implies that instead of incurring cost due to implementation of the controls, there will be some savings on the cost of implementation.

Fig. 4.14 shows the profile of the adjoint variable for the recovered class with respect to the three scenarios presented. It shows that the marginal cost associated with a unit change in the recovery class is either zero or reducing with time. From the graph, we observed that the cost is either zero or reducing with time. This implies that the implementation of either of the single control measures brings about decrease in the marginal cost of increasing the Recovered class population as time progresses while the concurrent implementation of both measures result in zero marginal cost for the recovered class. 


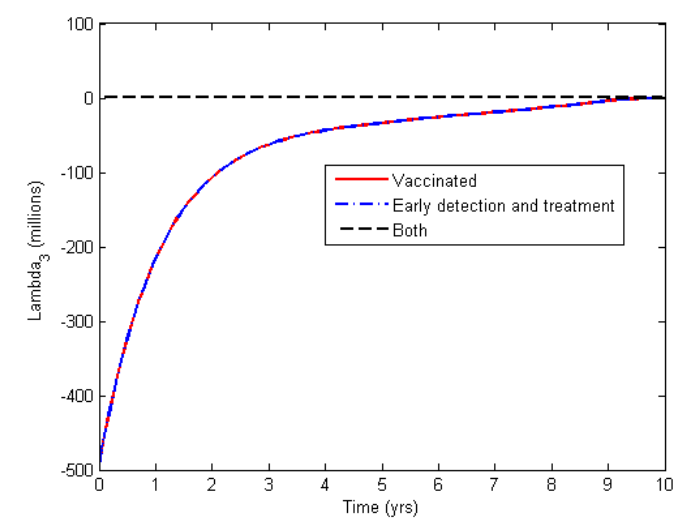

Fig.4.12 Adjointvariable $\lambda_{3}$

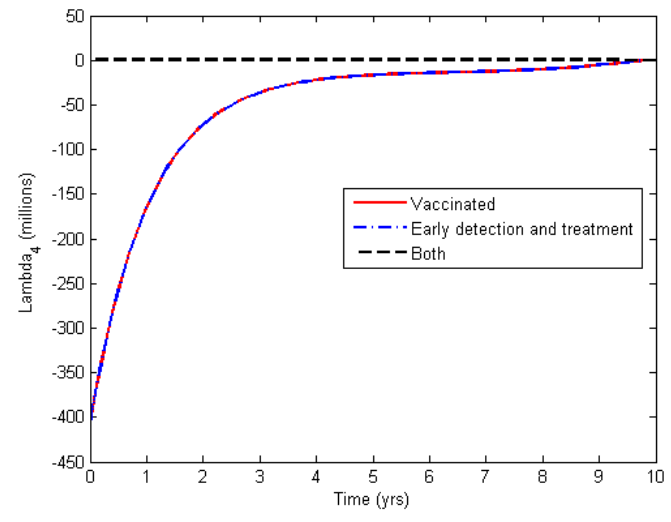

Fig. 4.13 Adjoint variable $\lambda_{4}$

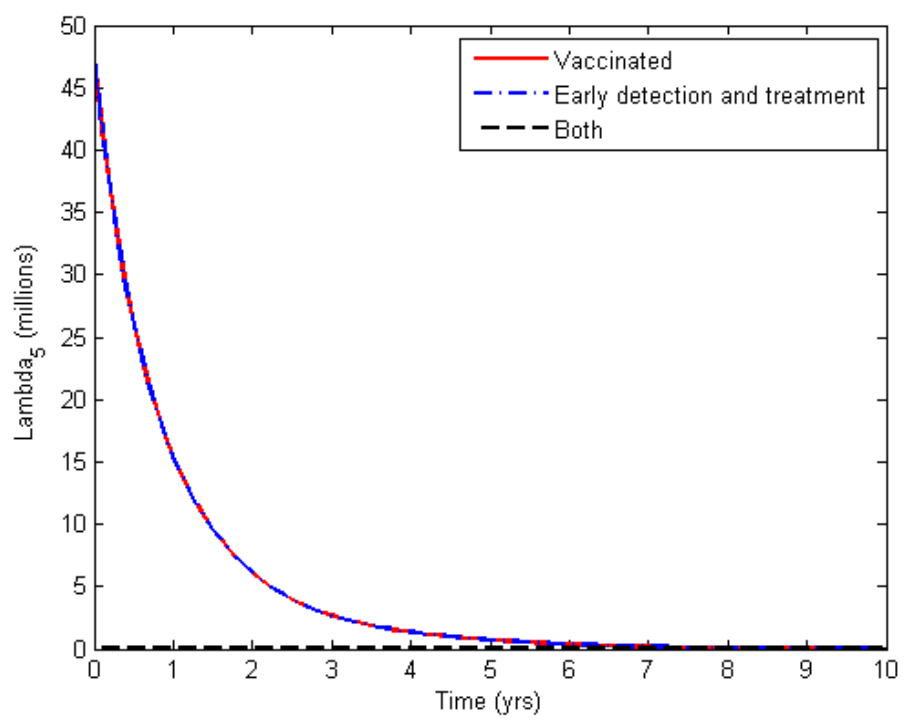

Fig. 4.14 Adjoint variable $\lambda_{5}$

\section{CONCLUSiON}

We presented a deterministic model for the spread and control of Hepatitis B virus infection. We derived the disease basic reproduction number $R_{0}$ and demonstrated that the situation under consideration is an endemic one $\left(\right.$ i.e. $\left.R_{0}>1\right)$. We reformulated the modelled situations as optimal control problem subject to our model dynamics with vaccination of the susceptibles and early detection cum treatment of the acutely-infected individuals as control measures.

The existence and uniqueness of the solution to the optimal control problem were established, the adjoint variable equations derived, and the control variables characterized based on Pontryagin's maximum principle. The resulting optimality system was solved numerically. Simulations of the numerical results showed that if the endemic situation is promptly addressed by implementing both control measures concurrently, there will be a remarkable reduction in the prevalence and incidence of the disease. Infact, the results showed that deploring the two control measures adequately could drive the endemic situation towards a disease free state within a decade.

\section{REFERENCES}

[1] Altekruse, S. F., McGlynn, K. A., Reichman, M. E. (2009). Hepato-cellular carcinoma incidence, mortality, and survival trends in the United States from 1975 to 2005. Journal of clinical oncolog, 27(9):1485-1491.

[2] Bailey, N. T. et al. (1975). The mathematical theory of infectious diseases and its applications. Charles Griffin \& Company Ltd, 5a Crendon Street, High Wycombe, Bucks HP13 6LE.

[3] Bakare, E. A., Nwagwo, A., and Danso- Addo, E. (2014). Optimal control analysis of an sir epidemic model with constant recruitment. International Journalof Applied Mathematical Research,3(3):273285. 
[4] Behncke, H. (2000). Optimal control of deterministic epidemics. Optimal Control Applications and methods, 21(2):269-285

[5] Edmunds, W., Medley, G., and Nokes, D. (1996). The traansmission dynamics and control of hepatitis b virus in the gambia. Statistics in medicine, 15(20):2215-2233.

[6] Fleming, W. H. and Rishel, R. W. (2012). Deterministic and stochastic optimal control, volume 1. Springer Science \& Business Media.

[7] Gaff, H. and Schaefer, E. (2009). Optimal control applied to vaccination and treatment strategies for various epidemiological models. Mathematical biosciences and engineering: MBE, 6(3):469-492.

[8] Greenhalgh, D. (1986). Control of an epidemic spreading in a heterogeneously mixing population. Mathematical biosciences, 80(2):23-45.

[9] Lenhart, S. and Workman, J. T. (2007). Optimal control applied to biological models. CRC press

[10] Parkin, D. M., Bray, F., Ferlay, T., and Pisani, P. (2005). Global cancer statistics, 2002. CA: a cancer for clinicicians, 55(2):74-108.

[11] [Schwartz, J. and Carithers, R. (2013). Epidemiology and etiologic associations of hepato- cellular carcinoma.

[12] Sethi, S. P. and Staats, P. W. (1978). Optimal control of some simple deterministic epidemic models. Journal of the Operational Research Society, pages 129-136.

[13] Venook, A. P., Papandreou, C., and de Guevara, L. L. (2010). The incidence and epidemiology of hepatocellular carcinoma: a global and regional perspective. Thee oncologist, 15(Supplement 4):5-13.

[14] WebMD (2005-2017). Hepatitis b.

[15] WHO (2017), Fact sheet on hepatits b infection.

[16] Wiah, E., Makinde, O., and Adetunde, I. (2015). Optimal control of hepatitis b virus disease in a population with infected immigrants. Engineering Mathematics Letters, 2015:Article-ID.

[17] Yusuf, T. T, and Benyah, F. (2012). Optimal control of vaccination and treatment for an sir epidemiological model. World Journal of Modeling and Simulation, 8(3):194-204.

[18] Yusuf, T., Fall, A., and Friedman, A. (2014). Strategies for controlling the spread of hepatitis b virus in an adult population. International Journal of Applied Scientific and Engineering Research, 5(11):1455-1469.

[19] Yusuf, T. T., and Benyah, F. (2011). Optimal strategy for controlling the spread of HIV/AIDS disease: a case study of South Africa. Journal of biological dynamics, 6(2):475-494.

\section{AUTHORS' BIOGRAPHY}

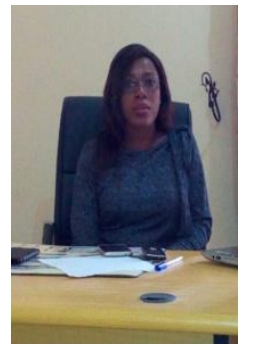

Ijalana Clara is a teaching assistant and a $\mathrm{PhD}$ student at the Department of Mathematical Sciences, Federal University of Technology, Akure, Nigeria.

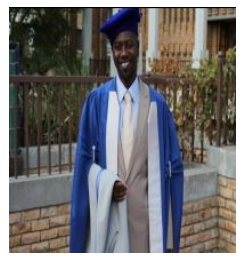

Dr. T.T Yusuf is a senior lecturer at the Department of Mathematical Sciences, Federal University of Technology, Akure, Nigeria

Citation: C. O. IJALANA, T. T. YUSUF, " Optimal Control Strategy for Hepatitis B Virus Epidemic in Areas of High Endemicity ", International Journal of Scientific and Innovative Mathematical Research, vol. 5, no. 12, p. 28-39, 2017., http://dx.doi.org/10.20431/2347-3142.0512003

Copyright: (C) 2017 Authors. This is an open-access article distributed under the terms of the Creative Commons Attribution License, which permits unrestricted use, distribution, and reproduction in any medium, provided the original author and source are credited. 